\title{
World cup soccer players tend to be born with sun and moon in adjacent zodiacal signs
}

\author{
J Verhulst
}

\begin{abstract}
The ecliptic elongation of the moon with respect to the sun does not show uniform distribution on the birth dates of the 704 soccer players selected for the 1998 World Cup. However, a uniform distribution is expected on astronomical grounds. The World Cup players show a very pronounced tendency $(p=0.00001)$ to be born on days when the sun and moon are in adjacent zodiacal signs.

(Br F Sports Med 2000;34:465-466)
\end{abstract}

Keywords: soccer; World Cup; astrology; moon

The dates of birth of the 704 players ( 22 players for each of the 32 national teams) selected for the World Cup (France 1998) have been published in the sport media. As there is controversy about the presence of astrological effects in sports champions, ${ }^{12}$ we decided to check this sample for possible astrological correlations.

The seasonal distribution of birth dates of top soccer players in several participating countries is strongly non-uniform, a selection effect induced by youth competition. ${ }^{34}$ This effect precludes the definition of a reliable null hypothesis for the distribution of zodiacal signs for the sun and planets (the zodiacal signs of the sun and planets such as Venus, Mars, and Jupiter are correlated for astronomical reasons).

Taken over a time span of some years, an approximately uniform distribution is expected for the moon signs. The moon travels through each of the 12 signs every month and, on average, spends about equal time in each. Therefore we decided to test the distribution of the zodiacal signs for the moon against this null hypothesis.

From an astronomical point of view, the zodiacal position of the moon is independent of the zodiacal position of the sun (to a very good approximation, knowledge of the sun sign does not permit a better than random prediction for the moon sign). However, astrologers state that the influence of a couple of luminaries changes as a function of the ecliptic elongation separating these luminaries (this relates to the supposed role of the so called "aspects" such as conjunction, opposition, trines or squares, which were especially cherished by Johannes Kepler). We therefore also decided to test the zodiacal elongation of the moon with respect to the sun for uniformity.

\section{Methods}

Because the hour of birth is not known for the 704 soccer players, we calculated the horoscopes at 12:00 Greenwich mean time on their day of birth. The ecliptic longitude of sun and moon were calculated to a precision of 1 minute, and the zodiacal sign of the sun and moon at that moment was recorded (no borderline cases occurred). It should be noted that the astrological signs do not correspond to the astronomical constellations along the ecliptic. The signs of astrology are obtained by dividing the zodiac into 12 sectors of 30 , starting at the vernal equinox.

For each horoscope, the zodiacal distance $\mathrm{z}$ was calculated. We define the zodiacal distance $\mathrm{z}$ as the number of sign boundaries or "cusps" that the sun has to traverse in order to move from its own sign into the moon sign. Thus, when sun and moon are in the same sign, $z=0$; when the two luminaries are in opposite signs, $z=6$; when the sun is in Aquarius and the moon in Capricorn, $z=11$, and so on. From an astronomical point of view, the zodiacal distance $z$ should show a uniform distribution.

\section{Results}

As predicted on astronomical grounds, the distribution of the moon signs does not differ significantly from uniformity $\left(\chi^{2}=10.34 ; \mathrm{df}=11\right.$; $\mathrm{p}=0.50$; table 1$)$. For the record, table 1 also gives the sign distribution for the sun. This distribution does not show any seasonal pattern,

Table 1 Distribution of the sun signs and moon signs on the day of birth (12:00 Greenwich mean time) of the 704 soccer players selected for the World Cup (France 1998)

\begin{tabular}{lll}
\hline Sign & Sun distribution & Moon distribution \\
\hline Aries & 72 & 53 \\
Taurus & 53 & 62 \\
Gemini & 44 & 68 \\
Cancer & 62 & 65 \\
Leo & 68 & 51 \\
Virgo & 62 & 70 \\
Libra & 55 & 59 \\
Scorpio & 61 & 55 \\
Sagittarius & 61 & 61 \\
Capricorn & 50 & 47 \\
Aquarius & 56 & 50 \\
Pisces & 60 & 63 \\
\hline
\end{tabular}


Table 2 Distribution of $z$ (first column; see text) for the 704 players selected for the World Cup (second column)

\begin{tabular}{ll}
\hline$z$ & $\begin{array}{l}\text { Cup players } \\
n=704\end{array}$ \\
\hline 0 & 52 \\
1 & 80 \\
2 & 59 \\
3 & 44 \\
4 & 75 \\
5 & 40 \\
6 & 45 \\
7 & 50 \\
8 & 61 \\
9 & 51 \\
10 & 59 \\
11 & 88 \\
\hline
\end{tabular}

and it does not differ significantly from a uniform distribution $\left(\chi^{2}=10.95 ; \mathrm{df}=11 ; \mathrm{p}=\right.$ $0.45)$. The absence of a seasonal pattern for the World Cup soccer players can be explained by the different regulations prevailing in the youth soccer competition of the participating countries. It is also possible that the selection effect $^{3}{ }^{4}$ is less important for very gifted youth players.

The distribution of $\mathrm{z}$ turns out to be strongly non-uniform (table $2 ; \chi^{2}=42.90 ; \mathrm{df}=11 ; \mathrm{p}=$ $0.00001)$. Multiplicative combination ${ }^{5}$ of the two $\mathrm{p}$ values considered $(0.50$ for the moon signs and 0.00001 for $\mathrm{z}$ ) yields an overarching $\mathrm{p}$ value of 0.00007 . We conclude that our result indicating the non-uniform distribution of the elongation values is significant.

\section{Discussion}

In our sample, the sun and moon tend to show up in adjacent signs $(z=1$ or 11$)$. Interestingly, the distribution of $\mathrm{z}$ seems to be symmetrical with respect to the conjunction-opposition axis (which corresponds to $z=0$ and 6 ). The populations for the $z$ values $1-2-3-4-5$ strongly correlate with the populations for the mirroring $\mathrm{z}$ values 11-10-9-8-7 (Spearman $\rho=1.000$; $\mathrm{p}<0.01)$. This observation is in accordance with the orthodox astrological view that symmetrical aspects (such as the two trine aspects of 120 and 240, or the two square aspects of 90 and 270) connecting a pair of luminaries have about the same significance for the native.

Although our results suggest that an astrological effect of some kind is involved, it proves nothing about the ability of astrologers to interpret horoscopes in any meaningful way. We even suspect that no astrologer would have been able to predict the outcome of the present investigation.

1 Benksi C, Caudron D, Galifret Y, et al. The "Mars Effect". A French test of over 1000 sports champions. Amherst, NY: Pro-

2 Ertel S. Mars effect uncovered in French sceptics' data. Correlation 1995;13:3-16.

3 Verhulst J. Seasonal birth distribution of West European soccer players: a possible explanation. Med Hypotheses 1992;38:346-8

4 Dudink A. Birth date and sporting success. Nature 1994;368:592.

5 Fisher RA. Statistical methods for research workers. 8th ed. Edinburgh: Oliver \& Boyd, 1941(Original work published in 1925).

\title{
British Association of Sport and Exercise Medicine in association with the National Sports Medicine Institute
}

\section{Education programme 2000/2001}

\author{
Current Concepts Meeting on Pre-event Screening \\ Churchill College, Cambridge
}

8-9 December 2000

\section{Injury Management and Medicine-Head, Neck and Upper Limb 11-16 February 2001 Lilleshall National Sports Centre}

For further details of these courses please contact Mr Barry Hill, The National Sports Medicine Institute, c/o Medical College of St Bartholomew's Hospital, Charterhouse Square, London EC1M 6BQ.

Tel 02072510583 (ext 237). Fax 0207251 0774. Email: barry.hill@nsmi.org.uk Web site: www.nsmi.org.uk 